\title{
Tetracycline attenuates calcifying nanoparticles-induced renal epithelial injury through suppression of inflammation, oxidative stress, and apoptosis in rat models
}

\author{
Yuqing Zhang ${ }^{1}$, Rujian Zhu ${ }^{1}$, Dong Liu ${ }^{1}$, Min Gong ${ }^{1}$, Wei Hu ${ }^{1}$, Qingtong Yi ${ }^{1}$, Jie Zhang ${ }^{2}$ \\ ${ }^{1}$ Department of Urology, Shanghai Pudong Hospital, Fudan University Pudong Medical Center, Shanghai 201399, China; ${ }^{2}$ School of Medicine, \\ Shanghai Jiao Tong University, Shanghai 200025, China \\ Contributions: (I) Conception and design: Y Zhang; (II) Administrative support: R Zhu; (III) Provision of study materials or patients: D Liu, W Hu; (IV) \\ Collection and assembly of data: Y Zhang, J Zhang; (V) Data analysis and interpretation: M Gong, Q Yi; (VI) Manuscript writing: All authors; (VII) \\ Final approval of manuscript: All authors. \\ Correspondence to: Rujian Zhu. Department of Urology, Shanghai Pudong Hospital, 2800 Gongwei Road, Shanghai 201399, China. \\ Email: tzzhurj@163.com.
}

Background: Calcifying nanoparticles (CNPs) has been associated with the occurrence and development
of kidney stones, but the exact mechanism is not clear. This study aimed to establish a rat model of CNP-
induced renal epithelial injury and assess the efficacy of tetracycline in preventing this injury.
Methods: Kidney stones from patients after percutaneous nephrolithotomy (PCNL) were collected to
isolate and culture CNPs. Thirty Sprague-Dawley rats were divided into three groups: the sham group (G1),
the CNP group (G2), and the CNP + tetracycline group (G3). Rats in G2 and G3 were given an intravenous
injection of CNPs via the tail vein, while rats in G1 were given saline. Meanwhile, rats in G3 were given
tetracycline by gavage twice a day at a dose of $25 \mathrm{mg} / \mathrm{kg}$. After 8 weeks, the 24-h urine of all rats was
collected, and all rats were sacrificed to obtain blood and kidneys.
Results: The results revealed that in G2, activities of antioxidant enzymes such as superoxide dismutase
and catalase were significantly lower than those in G1, while malondialdehyde activity in G2 was significantly
higher than that in G1 and both of them were inhibited by tetracycline co-treatment in G3. CNPs
significantly increased expression of inflammatory cytokines, including monocyte chemotactic protein 1 and
interleukin 6, which were largely alleviated in G3. CNPs significantly increased TUNEL-positive cells and
the apoptosis activity of Bcl2-associated X protein but decreased B-cell lymphoma-2 level compared with
that in G1, and was limited by tetracycline co-treatment in G3. Furthermore, CNPs led to notable renal
tubular epithelial cell damage, hyaline cast formation, desquamation, swelling, vacuolization in histology, all
of which were alleviated by tetracycline.

Conclusions: Tetracycline can attenuate CNP-induced renal epithelial injury through suppression of inflammation, oxidative stress, and apoptosis.

Keywords: Calcifying nanoparticles (CNPs); renal epithelial injury; tetracycline (TCN)

Submitted Aug 13, 2019. Accepted for publication Nov 05, 2019.

doi: $10.21037 /$ tau.2019.11.14

View this article at: http://dx.doi.org/10.21037/tau.2019.11.14

\section{Introduction}

The prevalence of kidney stones has increased from $1.16 \%$ to $7.54 \%$ in the past few decades in China (1) and continues to climb. On account of a high recurrence rate of up to
$50 \%$ within the subsequent 10 years after the first episode, the rate of repeated operative intervention is also very high (2). Due to a lack of satisfactory therapeutic options, these patients will have a lower quality of life compared 
with the general public (3). Therefore, there is an urgent need to identify novel therapeutic methods and develop effective strategies for treatment and prevention of kidney stones. Many investigators have found that renal epithelial cell injury increases the likelihood of crystal attachment both in vivo and in vitro (4). However, the exact mechanism of kidney stone formation has not yet been clarified.

Calcifying nanoparticles (CNPs), previously called "nanobacteria", were first proposed by Kajander et al. (5). The accumulated evidence failed to provide reasonable support for the existence of small microorganisms referred to as nanobacteria because of the ambiguous nature of the defining properties of nanobacteria and the lack of bacteriologic evidence of culturability (6). There are several characteristics of CNPs, including proliferation and replication by a cultured, heat-resistant apatite mineralized, biogenic shell on their membrane which can cause mineralization in vitro under physiologic concentrations of calcium and phosphate. They have been detected in numerous pathological calcifications in human diseases, especially in ectopic calcification, which may play a potentially key role in the pathogenesis of kidney stones $(5,7,8)$, polycystic kidney disease (PKD) (9), type III prostatitis (10), coronary artery calcification, and placental calcification (11). Furthermore, CNPs can be detected in the kidneys of patients with end-stage chronic kidney disease (CKD), whereas no particles can be detected in kidneys of healthy controls (12). CNPs were reported to cause renal tubular epithelial cell damage calcification by inducing chronic inflammation of the kidney (13). Interestingly, compared with the acute kidney injury model, CNPs are more likely to cause chronic injury of renal tubular epithelial cells and apoptosis, which leads to renal tubular calcification (14), and thus corresponds more with the chronic pathological process of kidney stones.

Tetracycline (TCN) has numerous pharmaceutical capacities including antibiotics, metal chelate, proteolysis, and iontophoresis. Furthermore, one study has shown that TCN can provide reactive oxygen species (ROS) scavenging and has anti-apoptotic properties and anti-inflammatory effects (15). TCN has been widely used in the treatment of osteoarthritis, periodontitis, dental stone formation, cancer metastasis, and offers protective effects on prion-mediated brain damage diseases $(16,17)$. TCN also has been proved to inhibit the multiplication of CNPs from both human kidney stones and kidney cyst fluids of patients with PKD (18).

In this study, we explored the underlying mechanisms of CNP-induced renal epithelium injury and whether the injury can be attenuated by TCN in vivo. We hope this can provide a potential novel treatment or prevention of kidney stones in the future.

\section{Methods}

\section{Cultivation of the CNPs}

The samples were collected from patients with kidney stones by nephrolithotomy surgery in the Department of Urology in Shanghai Pudong Hospital. In the analyses, all the stones we used were calcium-based stones of calcium oxalate and calcium phosphate. The study obtained informed consent from patients and was approved by the ethics committee (2016-PWQ-04). The stones were washed and crushed into small pieces, then demineralized with hydrochloric acid. After neutralization, filtration $(0.45 \mu \mathrm{m})$, and centrifugation, the bottom liquid was taken into RPMI-1640 medium (Gibco, CA, USA) containing $10 \%$ heat-inactivated $\gamma$-FBS (Gibco, CA, USA), and then placed into an incubator with $5 \% \mathrm{CO}_{2}$ at $37{ }^{\circ} \mathrm{C}$. The medium was replaced every 2 weeks and lasted for 8 weeks. All steps were strictly performed in a sterile laminar flow environment. The same serum and culture solution were added to the control group, and the culture was carried out under the same conditions, but no stone filtration solution was added.

\section{Characterization of the CNPs}

Transmission electron microscope (TEM) examination The cultured CNPs were fixed in 3\% glutaraldehyde at $4{ }^{\circ} \mathrm{C}$ for $3 \mathrm{~h}$, post-fixed in $1 \%$ osmic acid at room temperature for $2 \mathrm{~h}$, and dehydrated in a graded concentration series of ethanol and acetone. Finally, the CNPs were embedded in epoxy resin to prepare ultrathin sections. The ultrathin sections were stained with uranyl acetate and lead citrate and analyzed using a Hitachi TEM system (Hitachi, Tokyo, Japan).

\section{Gram staining}

The cultured CNPs were heat-fixed to the slide, stained with crystal violet dye for $10 \mathrm{~s}$, iodine solution for $10 \mathrm{~s}$, decolorized with alcohol for $10 \mathrm{~s}$, stained with safranin for $1 \mathrm{~min}$, and finally washed with a gentle stream of water.

\section{Alizarin red staining}

The cultured CNPs were heat-fixed to the slide, stained 
with alizarin red dye solution (G1038, Wuhan Servicebio Technology Company, Wuhan, China) for $10 \mathrm{~min}$, and finally washed with a gentle stream of water.

\section{von Kossa staining}

The cultured CNPs were heat-fixed to the slide, incubated with silver nitrate solution for $30 \mathrm{~min}$, exposed to ultraviolet light for $3 \mathrm{~h}$, stained with hematoxylin staining for $5 \mathrm{~min}$, and finally stained with eosin for $5 \mathrm{~min}$.

\section{Animal experimental design and animal tissue preparation}

Thirty male SD rats $(160-180 \mathrm{~g})$ were purchased from Shanghai SIPPR-BK Laboratory or Animal Co. Ltd. After 1 week of adaptive feeding, they were randomly divided into three groups of 10 rats each: the Sham group (G1), the CNP group (G2), and the CNP + TCN group (G3). Rats in the G2 and G3 were given an intravenous injection of CNPs (OD =2.0, $0.4 \mathrm{~mL}$ ) via the tail vein, while rats in G1 were given saline. Meanwhile, rats in G3 were given TCN (T829835; Shanghai Macklin Biochemical Institute, Shanghai, China) by gavage twice a day at a dose of 25 $\mathrm{mg} / \mathrm{kg}$. After 8 weeks, 24-h urine of all rats was collected, and the rats were then sacrificed to obtain the blood and kidneys. A portion of the kidneys was flash-frozen in liquid nitrogen while the rest were fixed in $4 \%$ paraformaldehyde. The study procedure was developed according to the guidelines of the ethics committee (2016-PWQ-04).

\section{Histological observation}

Kidneys were paraffin-embedded and sectioned into $4 \mu \mathrm{m}$ slices. The slides were deparaffinized in xylene and ethanol and then stained with hematoxylin and eosin (HE).

\section{Serum and urine biochemistry}

Serum blood urea nitrogen (BUN), uric acid (UA), creatinine $(\mathrm{Cr}), \mathrm{Ca}^{2+}, \mathrm{Mg}^{2+}$, and $\mathrm{P}^{3+}$ levels along with urine $\mathrm{Ca}^{2+}, \mathrm{Mg}^{2+}$, and $\mathrm{P}^{3+}$ levels were measured in strict accordance with the instructions of commercial assay kits for BUN, UA, Cr, $\mathrm{Ca}^{2+}, \mathrm{Mg}^{2+}$ and $\mathrm{P}^{3+}(\mathrm{C} 010, \mathrm{C} 075, \mathrm{C} 074$, C012, C015 and C014; Changchun Huili Bioengineering Company, Changchun, China).

\section{Oxidative stress assay}

The renal homogenate was used to measure levels of superoxide dismutase (SOD), catalase (CAT), and malondialdehyde (MDA) in strict accordance with the instructions of commercial assay kits for SOD, CAT, and MDA (A001-1, A007-1 and A003-1; Nanjing Jiancheng Bioengineering Institute, Nanjing, China) respectively. The protein concentration of the kidney homogenate was measured by BCA Protein Assay Kit (Thermo Fisher Scientific, MA, USA).

\section{Immunobistochemistry (IHC) assay}

The slides were deparaffinized in xylene and ethanol and then performed antigen retrieval with sodium citrate in a microwave oven. The slides were incubated with $3 \%$ hydrogen peroxide solution to block the endogenous peroxidase. All slides were then incubated for $30 \mathrm{~min}$ in goat serum, followed by overnight incubation at $4{ }^{\circ} \mathrm{C}$ with primary antibodies MCP-1 (ab25124, Abcam Inc., MA, USA) and IL-6 (ab9324, Abcam Inc., MA, USA). The next day, the slides were incubated with the second antibody at room temperature for $50 \mathrm{~min}$ and then incubated with $\mathrm{DAB}$. The dyeing time was controlled under a microscope, and the slice was washed with a gentle stream of water. Image-Pro Plus (Media Cybernetics, Rockville, MD, USA) was used to perform the quantitative analysis.

\section{Terminal dUTP nick end labeling (TUNEL) assay}

The slides were deparaffinized in xylene and ethanol, incubated with protease $\mathrm{K}$ working solution for $25 \mathrm{~min}$ at $37^{\circ} \mathrm{C}$, incubated with the solution for $20 \mathrm{~min}$ at room temperature to rupture membranes, incubated with $\mathrm{TdT}$ and dUTP mixture (1:9) for $2 \mathrm{~h}$ at $37{ }^{\circ} \mathrm{C}$, and finally incubated with DAPI for $10 \mathrm{~min}$ at room temperature. Between the above steps, the slides were washed 3 times with PBS for 5 min each time. The sections were observed under a fluorescence microscope.

\section{Western blot (WB) assay of Bax and Bcl-2}

The total protein of the renal cortex tissue was extracted, and the protein concentration was determined by BCA Protein Assay Kit (Thermo Fisher Scientific, MA, USA). Then, $20 \mu \mathrm{g}$ proteins were separated by using $10 \%$ SDSpolyacrylamide gel electrophoresis and transferred to the polyvinylidene difluoride membrane. Membranes were blocked with $5 \%$ skim milk for $1 \mathrm{~h}$ at room temperature. The membranes then were incubated at $4{ }^{\circ} \mathrm{C}$ overnight 

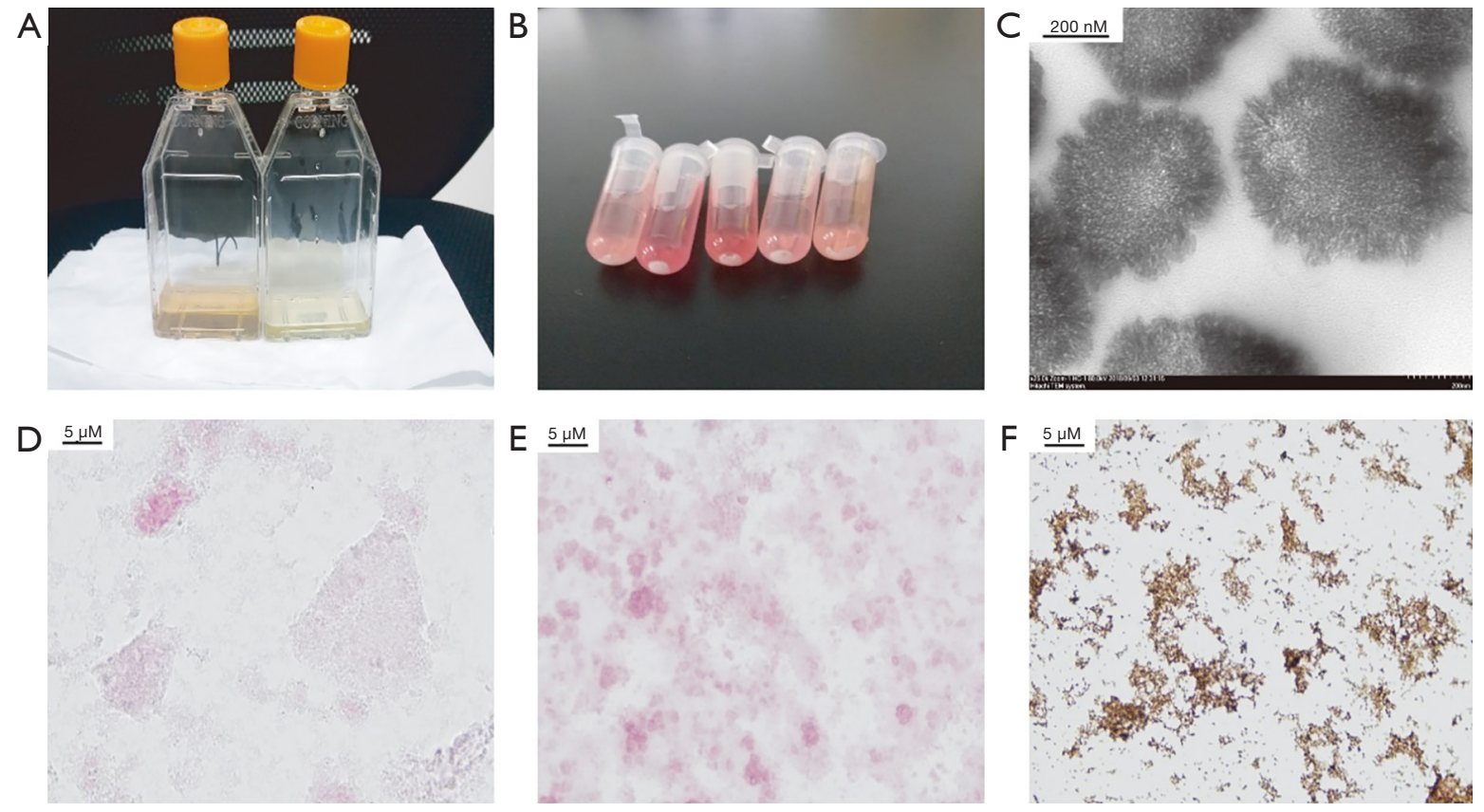

Figure 1 Characteristics of CNPs. (A) Visible mineralized biofilms on the right bottle after 6 weeks; (B) the white precipitate after centrifugation; (C) TEM showed that CNPs had irregularly spherical morphology with a dense, needle-like hydroxyapatite shell; (D) Gram staining of CNPs; (E) Alizarin red staining of CNPs; (F) von Kossa staining of CNPs. CNPs, calcifying nanoparticles; TEM, transmission electron microscope.

with primary antibody against Bax, $\beta$-actin (GB11001 and GB11007; Wuhan Servicebio Technology Company, Wuhan, China) or Bcl-2 (WL01556; Shenyang Wanlei Technology Company, Shenyang, China). The next day, the membranes were incubated with the appropriate secondary antibodies for $1 \mathrm{~h}$ at room temperature. Finally, immunoreactive protein bands were visualized using ECL (Thermo fisher scientific, MA, USA). The grey image analysis of immunoblots was performed by using ImagePro Plus.

\section{Statistical analysis}

Results are shown as mean \pm SD and were analyzed using SPSS 21.0 (IBM, Chicago, IL, USA). Differences between the two groups were assessed for significance using Student's $t$-test; $\mathrm{P}<0.05$ denoted statistical significance.

\section{Results}

\section{CNP characteristics (Figure 1)}

The culture flask of the CNP group appeared to have white precipitate adhering to the bottle wall after 4 weeks, and visible mineralized biofilms appeared after 6 weeks, while the control group showed no change (Figure 1A). The white precipitate was observed on the bottom of the tube after centrifugation (Figure 1B). TEM showed that CNPs had irregularly spherical morphology with a dense, needle-like hydroxyapatite shell and a size of about 100-500 $\mathrm{nm}$ (Figure 1C). The CNPs were Gram-negative particles (Figure 1D), and the alizarin red staining was orange-red and clustered (Figure 1E). Since the outer shell of CNPs contains apatite, von Kossa staining showed a large number of sphericalshaped tan particles distributed in a sheet (Figure 1F).

\section{TCN alleviates CNP-induced renal histological injury}

The effect of TCN co-treatment on CNP-induced renal pathological lesions was analyzed. Kidney tissues from G1 showed normal histological features; meanwhile, in G3, there were obvious pathological lesions in the renal cortex and medulla, characterized by loss of brush border, vacuolization, hyaline cast formation, and desquamation of tubular epithelial cells. However, a marked amelioration was observed in that TCN co-treatment alleviated CNP- 

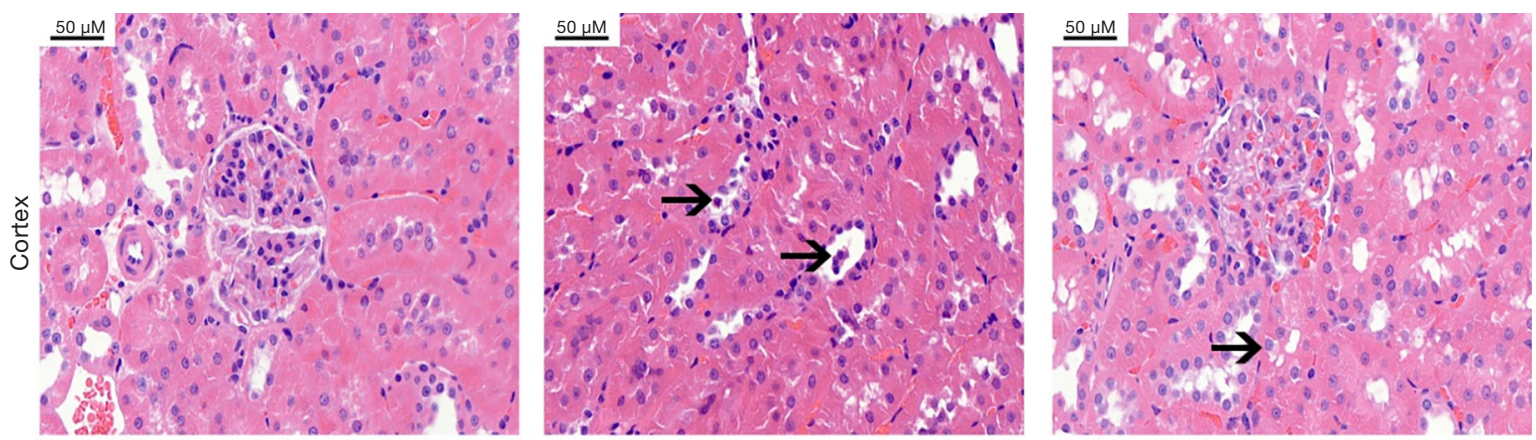

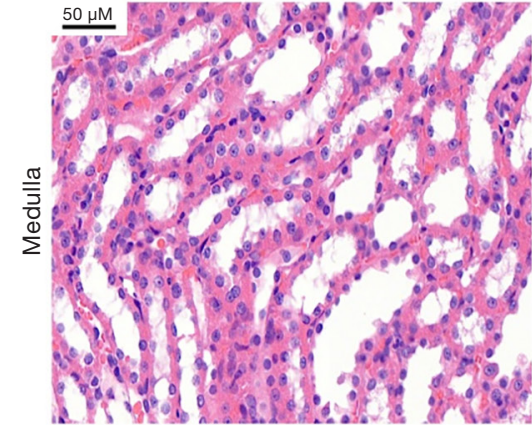

G1

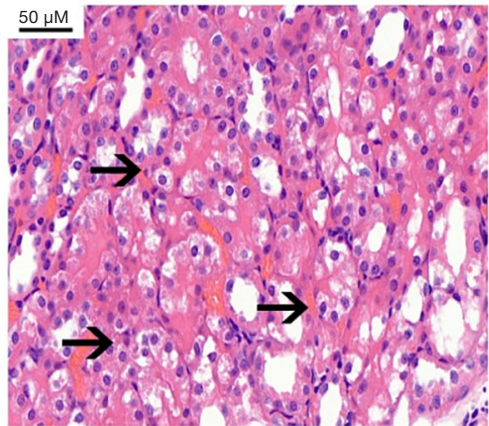

G2

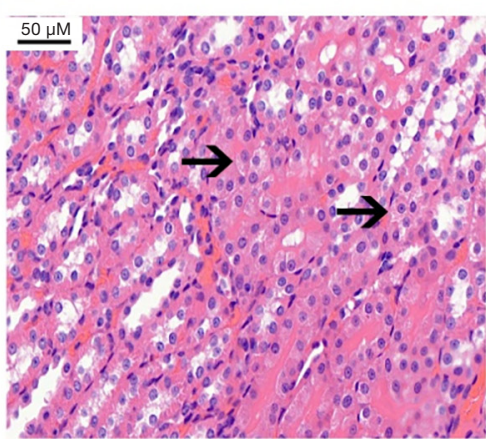

G3

Figure 2 Normal renal cortex and medulla histological features were observed in G1. Black arrows indicate the desquamation of tubular epithelial cells in the cortex and vacuolization in the medulla in G2. A marked amelioration was observed that tetracycline co-treatment alleviated CNP-induced renal histological injury in G3. G1: the sham group; G2: the CNP group; G3: the CNP + tetracycline group. CNP, calcifying nanoparticle.

Table 1 Urine biochemistry

\begin{tabular}{lccr}
\hline Group & $\mathrm{P}^{3+}(\mathrm{mmol} / \mathrm{L})$ & $\mathrm{Ca}^{2+}(\mathrm{mmol} / \mathrm{L})$ & $\mathrm{Mg}(\mathrm{mmol} / \mathrm{L})$ \\
\hline $\mathrm{G} 1$ & $6.55 \pm 3.20$ & $1.34 \pm 0.18$ & $1.14 \pm 0.15$ \\
G2 & $7.53 \pm 1.38$ & $1.63 \pm 0.23^{*}$ & $1.07 \pm 0.06$ \\
G3 & $8.08 \pm 0.60$ & $1.36 \pm 0.15^{\circ}$ & $1.02 \pm 0.08$ \\
\hline
\end{tabular}

*, compared with G1, P<0.05; ${ }^{\circ}$, compared with G2, P<0.05. G1: the sham group; G2: the CNP group; G3: the CNP + tetracycline group.

induced renal histological injury (Figure 2).

\section{Serum and urine biochemistry}

There was no statistical difference in urine volume between the rats in the three groups. The calcium level in $24 \mathrm{~h}$ urine from G2 was significantly higher than that from G1 and G3 $(\mathrm{P}<0.05)$. There were no statistical differences among the three groups in terms of $\mathrm{P}^{3+}$ and $\mathrm{Mg}^{2+}$ (Table 1). There were no statistical differences in the levels of $\mathrm{P}^{3+}, \mathrm{Ca}^{2+}, \mathrm{Mg}^{2+}$, BUN, UA, and $\mathrm{Cr}$ in the serum of rats (Table 2).

\section{TCN alleviates CNP-induced renal oxidative stress}

SOD and CAT activities were both significantly lower in G2 than that in G1. By contrast, the level of renal MDA was elevated in G2 compared to G1. These two sets of data were markedly reduced by TCN co-treatment (Table 3). The present study assessed the protective role of TCN cotreatment that relies on the alleviation of oxidative stress.

\section{TCN reduces CNP-induced tubular epithelial cell apoptosis}

TUNEL assays were performed to investigate the apoptosis 
Table 2 Serum biochemistry

\begin{tabular}{lcccccc}
\hline Group & $\mathrm{P}^{3+}(\mathrm{mmol} / \mathrm{L})$ & $\mathrm{Ca}^{2+}(\mathrm{mmol} / \mathrm{L})$ & $\mathrm{Mg}^{2+}(\mathrm{mmol} / \mathrm{L})$ & $\mathrm{BUN}(\mathrm{mg} / \mathrm{dL})$ & $\mathrm{UA}(\mathrm{umol} / \mathrm{L})$ & $\mathrm{Cr}(\mathrm{umol} / \mathrm{L})$ \\
\hline G1 & $2.25 \pm 0.13$ & $2.62 \pm 0.07$ & $0.9456 \pm 0.0095$ & $17.10 \pm 2.42$ & $132.40 \pm 9.05$ & $103.30 \pm 10.42$ \\
G2 & $2.21 \pm 0.13$ & $2.66 \pm 0.03$ & $0.9430 \pm 0.0088$ & $14.90 \pm 2.37$ & $126.60 \pm 12.60$ & $95.98 \pm 9.60$ \\
G3 & $2.30 \pm 0.33$ & $2.71 \pm 0.07$ & $0.9654 \pm 0.0450$ & $16.53 \pm 3.80$ & $117.10 \pm 24.96$ & $97.20 \pm 10.00$ \\
\hline
\end{tabular}

G1: the sham group; G2: the CNP group; G3: the CNP + tetracycline group. BUN, blood urea nitrogen; UA, uric acid; Cr, creatinine.

Table 3 Renal tissue oxidative stress index

\begin{tabular}{lcccc}
\hline Group & CAT $(\mathrm{U} / \mathrm{mg})$ & SOD $(\mathrm{U} / \mathrm{mg})$ & MDA $(\mathrm{nmol} / \mathrm{mg})$ & $\mathrm{BUN}(\mathrm{mg} / \mathrm{dL})$ \\
\hline G1 & $56.73 \pm 4.10$ & $554.40 \pm 50.44$ & $0.52 \pm 0.12$ & $17.10 \pm 2.42$ \\
G2 & $48.19 \pm 6.67^{\star}$ & $486.10 \pm 51.78^{\star}$ & $0.75 \pm 0.20^{\star}$ & $14.90 \pm 2.37$ \\
G3 & $55.69 \pm 9.26^{\circ}$ & $553.80 \pm 82.41^{\circ}$ & $0.55 \pm 0.13^{\circ}$ & $16.53 \pm 3.80$ \\
\hline
\end{tabular}

*, compared with $\mathrm{G} 1, \mathrm{P}<0.05$; ' , compared with $\mathrm{G} 2, \mathrm{P}<0.05$. G1: the sham group; G2: the CNP group; G3: the CNP + tetracycline group. CAT, catalase; SOD, superoxide dismutase; MDA, malondialdehyde.

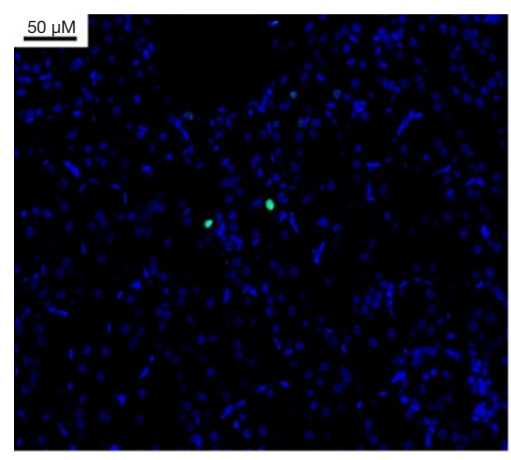

G1

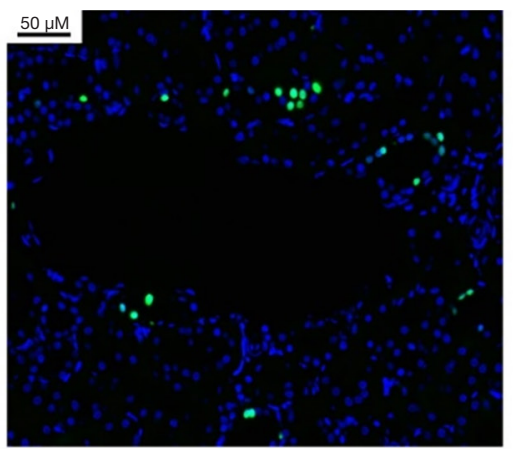

G2

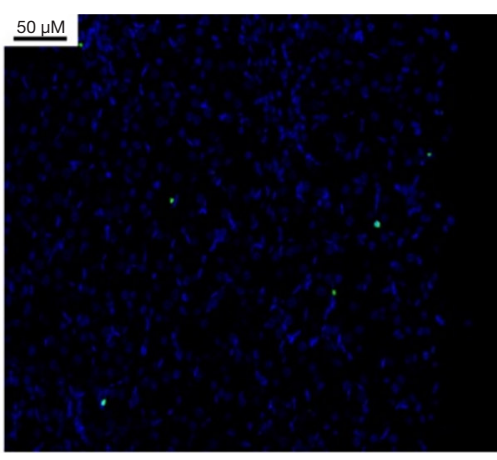

G3

Figure 3 Apoptosis in G1, G2, and G3. The apoptotic cells were stained with green colors. G1: the sham group; G2: the CNP group; G3: the CNP + tetracycline group.

level in the three groups. The results showed that the renal tissue apoptosis level in G2 was higher than that of G1 and G3 (Figure 3). WB assay indicated that the protein expression of apoptosis marker Bax in G2 was significantly higher than that in G1 and G3, and the protein expression of apoptosis marker Bcl-2 was remarkably lower than that in G1 and G3 (Figure 4). It was verified that TCN cotreatment prohibited CNP-induced renal cell apoptosis through WB assay and TUNEL assay, respectively.

\section{TCN suppressed renal inflammation induced by CNPs}

IHC demonstrated that the expression of inflammation markers (MCP-1 and IL-6) in the kidneys of G2 were higher than that of G1 and G3. In addition, TCN rescued high inflammation caused by CNPs (Figure 5). The antiinflammatory property of TCN co-treatment through suppressing renal MCP-1, IL-6 expression was clearly observed.

\section{Discussion}

Worldwide, nephrolithiasis has a high incidence, high recurrence rate, and a high cost of treatment. New approaches to prevent and treat kidney stones could significantly reduce costs to government healthcare systems and increase the quality of life. However, the understanding of the mechanisms of kidney stone formation and growth is 

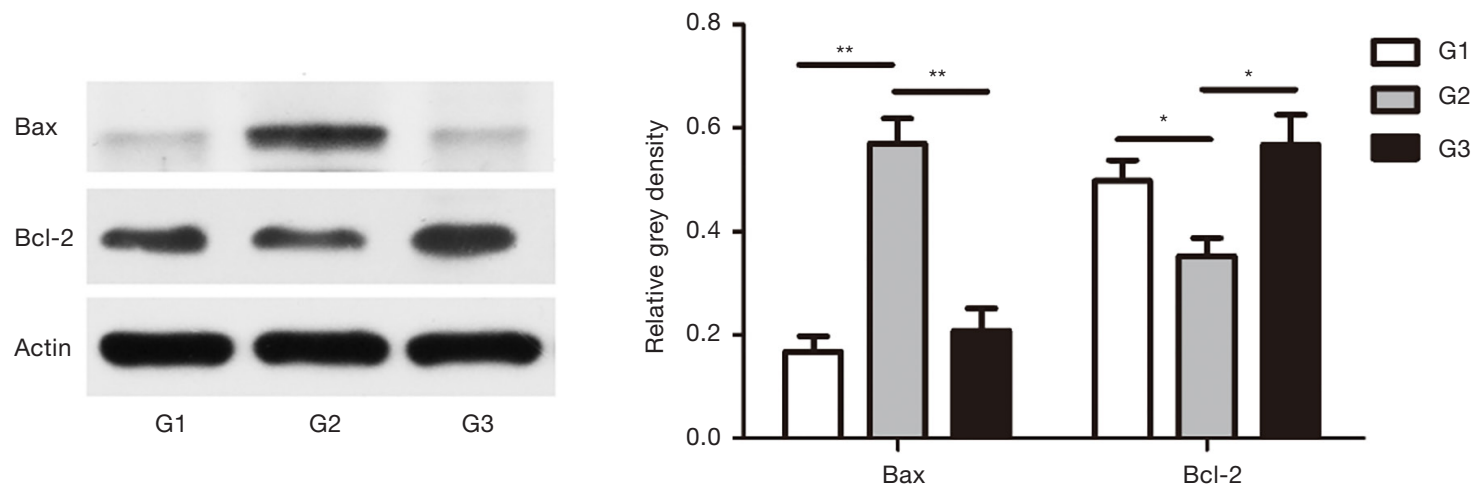

Figure 4 Expressions of Bax and Bcl-2 in kidneys of rats detected by Western blot. *, $\mathrm{P}<0.05$; **, $\mathrm{P}<0.01$. G1: the sham group; G2: the CNP group; G3: the CNP + tetracycline group.

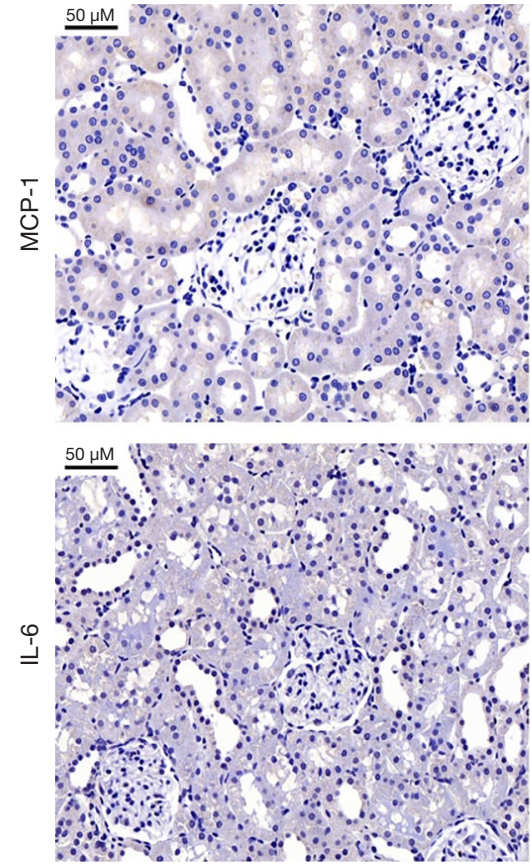

G1
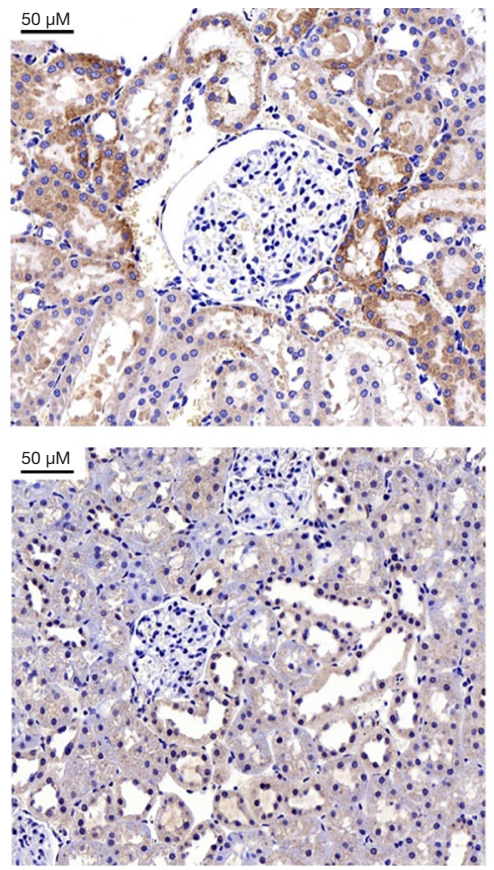

G2
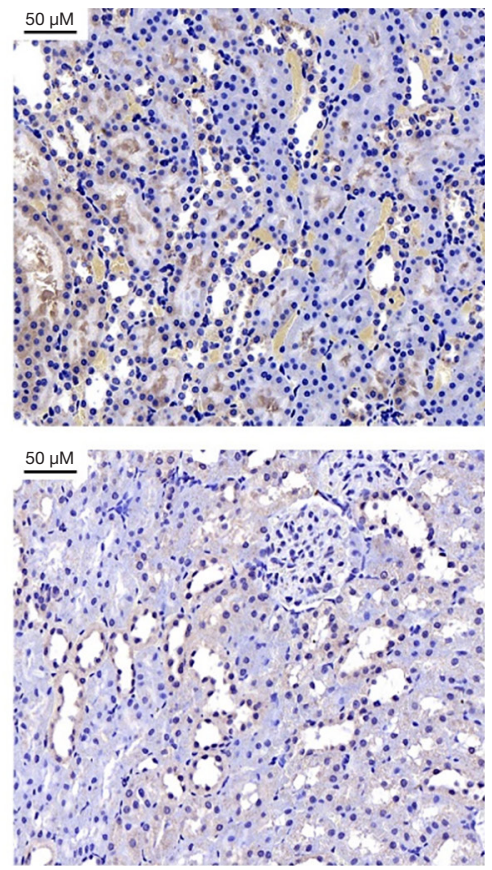

G3

Figure 5 Expressions of MCP-1 and IL-6 in kidney tissues, IHC staining. MCP-1 protein is mainly distributed in the cytoplasm of renal tubular epithelial cells and mesangial cells. IL-6 protein is mainly distributed in the cytoplasm of renal tubular epithelial cells. G1: the sham group; G2: the CNP group; G3: the CNP + tetracycline group.

still limited.

A bulk of experimental and clinical observations strongly suggest that crystals do not adhere to the normally differentiated tubular epithelium, but rather attach to dedifferentiated/regenerating epithelial cells $(19,20)$ and the luminal surface of the tubular epithelium under stress conditions. Crystal-binding molecules usually are not present at the luminal membrane of intact differentiated tubular epithelia (21). Bellini duct (BD) plugs theory asserts that the renal tubule epithelium can be destroyed completely on account of papillary injury from the BD plug so that crystals can attach to the tubular basement membranes (22). Crystalluria can be detected in healthy individuals while stone formation is not as common as crystalluria. Crystals are more likely to attach to a site of injury in the nephron in certain individuals (23). Renal 
epithelial injury is the basis for the interaction between crystals and cells, and the interaction of crystal and cells will further aggravate tubular cell damage that may eventually result in the retention of crystals. The increasing crystal attachment after the injury is due to the molecules exposed to the cell surfaces that are not normally accessible to crystals. Crystal adherence to components of the exposed basement membrane may contribute to crystal retention in the kidney, and then-nascent crystals will be retained in this place to form calculi in certain individuals (24).

As a novel material, CNPs have been discussed for more than two decades since first described by Kajander and Ciftcioglu (5). CNPs are mineral-protein nanoparticles with biomimetic functions and are closely related to various pathological calcification processes in the body (25). CNPs exist in blood, urine, and other organs or tissues, and tend to accumulate in the kidneys (26). Hu et al. found exposing HK-2 cells to CNPs led to swelling and vacuolization of mitochondria, and CNPs decreased mitochondrial membrane potential, resulting in mitochondrial damage and further eventual damage to other cellular components (27). There seems to be a special cell adhesion molecule in the outer membrane of the CNPs that may impair the integrated renal epithelial cells. The same mechanism has been mentioned in dental disease caused by CNPs (28).

There is evidence of a link between CNPs and the presence of Randall's plaques. Kumar et al. suggested a probable role of CNPs in the etiopathogenesis of Randall's plaques that are precursors of calcium oxalate kidney stones (29). In another study, Ciftçioğlu et al. detected CNPs in 70-90\% of kidney papillae samples with Randall's plaques while conversely noted that more than $80 \%$ of papillae samples without Randall's plaques were free of CNPs (30). Khan et al. reported that sub-lethal injury or dysfunctional cells might produce ineffective crystallization modulators and localized areas of supersaturation in the interstitium, which in turn may cause precipitation in the interstitium and development of Randall's plaques (31). Our study has verified that CNP induces renal injury, and this may explain the correlation between Randall's plaques and the presence of CNP.

TCN and its semisynthetic compounds have been proved to have both antibiotic and nonantibiotic properties in recent experiments. Besides acting as an antibiotic, TCN may also has metal chelate, proteolysis, and iontophoresis effects. It has been demonstrated that minocycline is more efficient than hydroxychloroquine in the treatment of early seropositive rheumatoid arthritis (32). Minocycline was also shown to effectively prevent the decrease in bone mineral density and trabecular bone through its dual effects on bone resorption and formation in ovariectomized osteoporosis rats (33). Application of doxycycline in deep periodontal sites can be considered as a justified approach for chronic periodontitis (34). Doxycycline can modify polymorphonuclear leucocyte activity in patients with acute myocardial infarction (35). Interestingly, CNPs can be detected at some stage in the pathological process of these diseases (36-38), indicating a correlation between these diseases and CNPs. It has also been reported that CNP levels and symptoms of CNP-associated interstitial cystitis/ painful bladder syndrome decreased dramatically after TCN treatment (39). Additional studies should be performed to elucidate the molecular mechanisms involved in CNP growth.

In this study, we verified CNP-induced renal epithelial injury in rats and examined the treatment effects of TCN. The results of CNP culture and morphological identification were similar to those of previous studies (40). CNPs caused obvious pathological lesions in the renal cortex and medulla of rats in G2, characterized by the loss of brush border, proximal convoluted tubular epithelial cellar damage, hyaline cast formation, desquamation of tubular epithelial cells, which is consistent with previous studies (41). Different from acute renal injury models like renal ischemia-reperfusion injury, CNP-induced injury is more likely to be a chronic, continuous process, which makes it reasonable to assume it mimics the pathophysiological processes of kidney stones, chronic nephrosis, and PKD.

Previous studies have indicated that oxidative stress could play a significant role in both renal epithelial injury and the development of kidney stones $(42,43)$. ROS overproduction results in lipid peroxidation, DNA mutation, apoptosis, necrosis, and finally causes endothelial cell and renal tubular epithelial cell injury (44). SOD and CAT activity, along with MDA content, are the representative events that reflect the oxidative status of tissues, lipid peroxidation, and cell injury (45). Thus, we examined the SOD and CAT activity and MDA concentration in kidney tissues of the three different groups. In the present study, the renal activity of self-protective enzymes including CAT and SOD activity, decreased while MDA increased in G2, indicating lipid peroxidation in kidneys. Our results also demonstrated that TCN could alleviate lipid peroxidation induced by CNPs.

Bcl-2 shows a pivotal role in preserving mitochondrial integrity, improving renal dysfunction, and suppressing tubular cell apoptosis (46). There is also evidence showing 


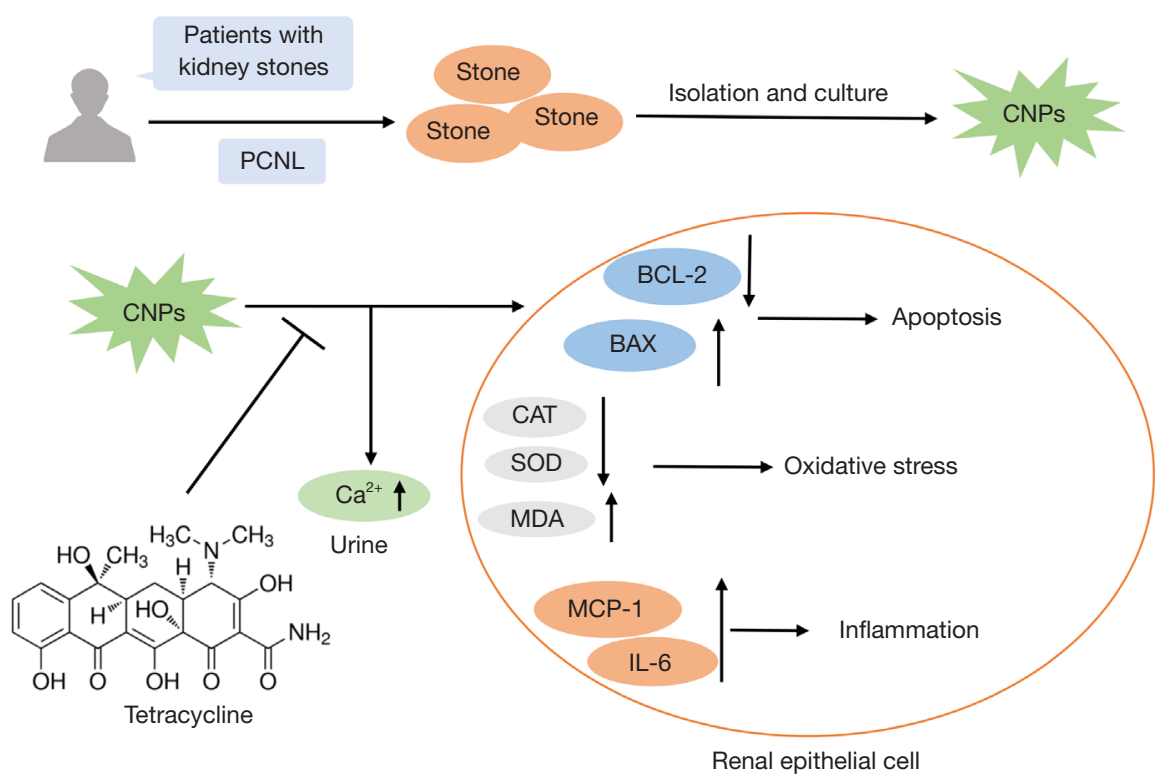

Figure 6 CNP-induced renal epithelial injury can be attenuated by TCN through suppression of inflammation, oxidative stress, and apoptosis. PCNL, percutaneous nephrolithotomy; CNPs, calcifying nanoparticles; CAT, catalase; SOD, superoxide dismutase; MDA, malondialdehyde; TCN, tetracycline.

that Bcl-2 inhibits lipid peroxidation through free-radical scavenging properties in vitro (47). In contrast, Bax can reverse these events and induce apoptosis (48). Our results demonstrated that TCN ameliorated apoptosis induced by CNPs.

The inflammatory reaction results in the production of multiple pro-inflammatory cytokines and chemokines like MCP-1 and IL-6 that activate and recruit leukocytes (49). CNPs have been verified to be a pro-inflammatory factor: they contribute to pathological processes once they aggregate and form larger mineral particles (50). Peng et al. injected CNPs into the peritoneal cavity of mice and found that the CNPs could cause pro-inflammatory responses in vivo (41). As a pro-inflammatory cytokine, IL-6 enhances the degree of renal injury, dysfunction, and inflammation by promoting the expression of adhesion molecules and subsequent oxidative stress (51). MCP-1 regulates migration and infiltration of monocytes/macrophages and has been demonstrated to be involved in response to inflammation induced by various diseases (52). Our present study showed that MCP-1 and IL-6 were up-regulated after CNP injection, while the treatment of TCN clearly suppressed there expression.

We also found hypercalciuria in the CNP group. Recent studies have shown that most calcium oxalate stones occurring in individuals with idiopathic hypercalciuria grow attached to Randall's plaque of calcium phosphate exposed to damaged renal papilla (23). Our study proved that CNPs might result in a hypercalciuric effect. It further explains why hypercalciuria differs across individuals, and this may be related to whether an individual carries CNPs. Meanwhile, other biochemical indicators and blood renal function indicators were not statistically different. A possible reason for this is that, in early-stage kidney injury, the glomerular filtration rate (GFR) is usually $\geq 50 \%$ of the normal value, and while the rates of increase in BUN and serum $\mathrm{Cr}$ (SCr) do not parallel the fall in GFR in a time frame (53), BUN and SCr concentrations do not increase rapidly. Also, SCr concentration does not reflect early kidney damage. We consider that kidney injury caused by CNPs is a chronic, progressive pathophysiological change that does not cause changes in serum renal function in a short period of time. Further research is needed to verify this point.

In summary, TCN attenuated CNP-induced renal epithelial injury through suppression of inflammation, oxidative stress, and apoptosis (Figure 6). This study illuminates the potential protective molecular mechanism of TCN in renal epithelial injury caused by CNPs and provides evidence for a TCN-based therapeutic strategy for 
future treatment of kidney stones and other pathological states associated with CNPs.

\section{Acknowledgments}

The authors would like to thank Bingqing Zou from Wuxi People's Hospital for help with the pathology section of this article.

Funding: The research was supported by a foundation of the Health and Family Planning Committee of Pudong New Area (PW2016B-16) and Key Discipline Construction Project of Pudong Health Bureau of Shanghai (PWZxk2017-21).

\section{Footnote}

Conflicts of Interest: The authors have no conflicts of interest to declare.

Ethical Statement: The authors are accountable for all aspects of the work in ensuring that questions related to the accuracy or integrity of any part of the work are appropriately investigated and resolved. This study has been approved by the Ethics Committee of Shanghai Pudong Hospital, Fudan University (2016-PWQ-04). Informed consent has been obtained from all patients.

\section{References}

1. Wang W, Fan J, Huang G, et al. Prevalence of kidney stones in mainland China: A systematic review. Sci Rep 2017;7:41630.

2. Galvin DJ, Pearle MS. The contemporary management of renal and ureteric calculi. BJU Int 2006;98:1283-8.

3. Parr JM, Desai D, Winkle D. Natural history and quality of life in patients with cystine urolithiasis: a single centre study. BJU Int 2015;116 Suppl 3:31-5.

4. Joshi S, Clapp WL, Wang W, et al. Osteogenic changes in kidneys of hyperoxaluric rats. Biochim Biophys Acta 2015;1852:2000-12.

5. Kajander EO, Ciftcioglu N. Nanobacteria: an alternative mechanism for pathogenic intra- and extracellular calcification and stone formation. Proc Natl Acad Sci U S A 1998;95:8274-9.

6. Cisar JO, Xu DQ, Thompson J, et al. An alternative interpretation of nanobacteria-induced biomineralization. Proc Natl Acad Sci U S A 2000;97:11511-5.

7. Kumon H, Matsumoto A, Uehara S, et al. Detection and isolation of nanobacteria-like particles from urinary stones: long-withheld data. Int J Urol 2011;18:458-65.

8. Khullar M, Sharma SK, Singh SK, et al. Morphological and immunological characteristics of nanobacteria from human renal stones of a north Indian population. Urol Res 2004;32:190-5.

9. Hjelle JT, Miller-Hjelle MA, Poxton IR, et al. Endotoxin and nanobacteria in polycystic kidney disease. Kidney Int 2000;57:2360-74.

10. Zhou Z, Hong L, Shen X, et al. Detection of nanobacteria infection in type III prostatitis. Urology 2008;71:1091-5.

11. Bertazzo S, Gentleman E, Cloyd KL, et al. Nanoanalytical electron microscopy reveals fundamental insights into human cardiovascular tissue calcification. Nat Mater 2013;12:576-83.

12. Wong TY, Wu CY, Martel J, et al. Detection and characterization of mineralo-organic nanoparticles in human kidneys. Sci Rep 2015;5:15272.

13. Shiekh FA, Miller VM, Lieske JC. Do calcifying nanoparticles promote nephrolithiasis? A review of the evidence. Clin Nephrol 2009;71:1-8.

14. Shiekh FA, Khullar M, Singh SK. Lithogenesis: induction of renal calcifications by nanobacteria. Urol Res 2006;34:53-7.

15. Griffin MO, Fricovsky E, Ceballos G, et al. Tetracyclines: a pleitropic family of compounds with promising therapeutic properties. Review of the literature. Am J Physiol Cell Physiol 2010;299:C539-48.

16. Zink MC, Uhrlaub J, DeWitt J, et al. Neuroprotective and anti-human immunodeficiency virus activity of minocycline. JAMA 2005;293:2003-11.

17. Forloni G, Iussich $S$, Awan $T$, et al. Tetracyclines affect prion infectivity. Proc Natl Acad Sci U S A 2002;99:10849-54.

18. Ciftcioglu N, Miller-Hjelle MA, Hjelle JT, et al. Inhibition of nanobacteria by antimicrobial drugs as measured by a modified microdilution method. Antimicrob Agents Chemother 2002;46:2077-86.

19. Gambaro G, Valente ML, Zanetti E, et al. Mild tubular damage induces calcium oxalate crystalluria in a model of subtle hyperoxaluria: Evidence that a second hit is necessary for renal lithogenesis. J Am Soc Nephrol 2006;17:2213-9.

20. Asselman M, Verhulst A, De Broe ME, et al. Calcium oxalate crystal adherence to hyaluronan-, osteopontin-, and CD44-expressing injured/regenerating tubular epithelial cells in rat kidneys. J Am Soc Nephrol 
2003;14:3155-66.

21. Vervaet BA, Verhulst A, D'Haese PC, et al. Nephrocalcinosis: new insights into mechanisms and consequences. Nephrol Dial Transplant 2009;24:2030-5.

22. Evan AP, Worcester EM, Coe FL, et al. Mechanisms of human kidney stone formation. Urolithiasis 2015;43 Suppl 1:19-32.

23. Evan AP. Physiopathology and etiology of stone formation in the kidney and the urinary tract. Pediatr Nephrol 2010;25:831-41.

24. Verkoelen CF, van der Boom BG, Houtsmuller AB, et al. Increased calcium oxalate monohydrate crystal binding to injured renal tubular epithelial cells in culture. Am J Physiol 1998;274:F958-65.

25. Wood HM, Shoskes DA. The role of nanobacteria in urologic disease. World J Urol 2006;24:51-4.

26. Martel J, Peng HH, Young D, et al. Of nanobacteria, nanoparticles, biofilms and their role in health and disease: facts, fancy and future. Nanomedicine (Lond) 2014;9:483-99.

27. Hu WG, Wang XF, Xu T, et al. Establishment nephrolithiasis rat model induced by nanobacteria and analysis of stone formation. Beijing Da Xue Xue Bao Yi Xue Ban 2010;42:433-5.

28. Yang F, Zeng J, Zhang W, et al. Evaluation of the interaction between calcifying nanoparticles and human dental pulp cells: a preliminary investigation. Int J Nanomedicine 2010;6:13-8.

29. Kumar V, Farell G, Yu S, et al. Cell biology of pathologic renal calcification: contribution of crystal transcytosis, cell-mediated calcification, and nanoparticles. J Investig Med 2006;54:412-24.

30. Ciftçioglu N, Björklund M, Kuorikoski K, et al. Nanobacteria: an infectious cause for kidney stone formation. Kidney Int 1999;56:1893-8.

31. Khan SR. Renal tubular damage/dysfunction: key to the formation of kidney stones. Urol Res 2006;34:86-91.

32. O'Dell JR, Blakely KW, Mallek JA, et al. Treatment of early seropositive rheumatoid arthritis: a twoyear, double-blind comparison of minocycline and hydroxychloroquine. Arthritis Rheum 2001;44:2235-41.

33. Williams S, Wakisaka A, Zeng QQ, et al. Effect of minocycline on osteoporosis. Adv Dent Res 1998;12:71-5.

34. Wennstrom JL, Newman HN, MacNeill SR, et al. Utilisation of locally delivered doxycycline in non-surgical treatment of chronic periodontitis. A comparative multi-centre trial of 2 treatment approaches.
J Clin Periodontol 2001;28:753-61.

35. Takeshita S, Ono Y, Kozuma K, et al. Modulation of oxidative burst of neutrophils by doxycycline in patients with acute myocardial infarction. J Antimicrob Chemother 2002;49:411-3.

36. Tsurumoto T, Matsumoto T, Yonekura A, et al. Nanobacteria-like particles in human arthritic synovial fluids. J Proteome Res 2006;5:1276-8.

37. Sommer AP. Could reduced bone mineral densities in HIV be caused by nanobacteria? J Proteome Res 2004;3:670-2.

38. Demir T. Is there any relation of nanobacteria with periodontal diseases? Med Hypotheses 2008;70:36-9.

39. Sapadin AN, Fleischmajer R. Tetracyclines: nonantibiotic properties and their clinical implications. J Am Acad Dermatol 2006;54:258-65.

40. Young JD, Martel J. The rise and fall of nanobacteria. Sci Am 2010;302:52-9.

41. Peng HH, Liu YJ, Ojcius DM, et al. Mineral particles stimulate innate immunity through neutrophil extracellular traps containing HMGB1. Sci Rep 2017;7:16628.

42. Huang HS, Ma MC. High Sodium-Induced Oxidative Stress and Poor Anticrystallization Defense Aggravate Calcium Oxalate Crystal Formation in Rat Hyperoxaluric Kidneys. PLoS One 2015;10:e0134764.

43. Fishman AI, Green D, Lynch A, et al. Preventive effect of specific antioxidant on oxidative renal cell injury associated with renal crystal formation. Urology 2013;82:489. e1-7.

44. Andreoli SP, McAteer JA. Reactive oxygen moleculemediated injury in endothelial and renal tubular epithelial cells in vitro. Kidney Int 1990;38:785-94.

45. Lu X, Wang C, Liu B. The role of Cu/Zn-SOD and MnSOD in the immune response to oxidative stress and pathogen challenge in the clam Meretrix meretrix. Fish Shellfish Immunol 2015;42:58-65.

46. Lindsten T, Ross AJ, King A, et al. The combined functions of proapoptotic Bcl-2 family members bak and bax are essential for normal development of multiple tissues. Mol Cell 2000;6:1389-99.

47. Hockenbery DM, Oltvai ZN, Yin XM, et al. Bcl-2 functions in an antioxidant pathway to prevent apoptosis. Cell 1993;75:241-51.

48. Jassem W, Fuggle SV, Rela M, et al. The role of mitochondria in ischemia/reperfusion injury. Transplantation 2002;73:493-9.

49. Bonventre JV, Zuk A. Ischemic acute renal failure: an 
inflammatory disease? Kidney Int 2004;66:480-5.

50. Peng HH, Wu CY, Young D, et al. Physicochemical and biological properties of biomimetic mineralo-protein nanoparticles formed spontaneously in biological fluids. Small 2013;9:2297-307.

51. Patel NS, Chatterjee PK, Di Paola R, et al. Endogenous interleukin-6 enhances the renal injury, dysfunction,

Cite this article as: Zhang $\mathrm{Y}$, Zhu R, Liu D, Gong M, Hu W, Yi Q, Zhang J. Tetracycline attenuates calcifying nanoparticlesinduced renal epithelial injury through suppression of inflammation, oxidative stress, and apoptosis in rat models. Transl Androl Urol 2019;8(6):619-630. doi: 10.21037/ tau.2019.11.14 and inflammation caused by ischemia/reperfusion. J

Pharmacol Exp Ther 2005;312:1170-8.

52. Deshmane SL, Kremlev S, Amini S, et al. Monocyte chemoattractant protein-1 (MCP-1): an overview. J Interferon Cytokine Res 2009;29:313-26.

53. Molitoris BA. Measuring glomerular filtration rate in acute kidney injury: yes, but not yet. Crit Care 2012;16:158. 